\title{
SOME MORE RESULTS ON R-NORM INFORMATION MEASURE
}

\author{
SATISH KUMAR
}

\begin{abstract}
In the present communication, I have defined the new information measure called " $\alpha$ - $R$-norm information measure". It has been characterized using infimum oiperation in Section 2 and axiomatically in Section 3 . Its properties have been studied in Section 4 , joint and conditional $\alpha$-R-norm information measure are studied in Section 5.
\end{abstract}

\section{Introduction}

Bockee and Lubbe [5] studied R-norm information measure of a discrete probability distribution

$$
P=\left(p_{1}, p_{2}, \ldots, p_{n}\right), \quad p_{i} \geq 0, i=1,2, \ldots, n
$$

where

$$
\sum_{i=1}^{n} p_{i}=1
$$

and

$$
R^{*}=\{R: R>0, R \neq 1\}
$$

given by

$$
H_{R}(P)=\frac{R}{R-1}\left[1-\left(\sum_{i=1}^{n} p_{i}^{R}\right)^{\frac{1}{R}}\right]
$$

The R-norm information measure (1.1) is a real function $\Delta_{n} \rightarrow R^{+}$, defined on $\Delta_{n}$ where $n \geq 2$ and $R^{+}$is the set of positive real numbers. This measure is different from Shannon's entropy [9], Renyi [8] and Havrda and Charvat [7] and Daroczy [6].

The most interesting property of this measure is that when $R \rightarrow 1$, R-norm information measure (1.1) approaches to Shannon's entropy and in case $R \rightarrow \infty, H_{R}(P) \rightarrow\left(1-\max p_{i}\right)$, $i=1,2, \ldots, n$.

The measure (1.1) has been generalized by Hooda and Anant [2] as:

$$
H_{R}^{\beta}(P)=\frac{R}{R+\beta-2}\left[1-\left(\sum_{i=1}^{n} p_{i}^{\frac{R}{2-\beta}}\right)^{\frac{2-\beta}{R}}\right], \quad 0<\beta \leq 1, R(>0) \neq 1
$$

Received February 27, 2008; revised July 25, 2008.

Key words and phrases. Concave function, functional equation, joint and canditional $\alpha$-R-norm information measure, operation infimum, Non-additivity, R-norm entropy. 
(1.2) has been called as the generalized R-norm entropy if degree $\beta$ which reduces to (1.1) when $\beta=1$. In case $R=1$, (1.2) reduces to

$$
H_{1}^{\beta}(P)=\frac{1}{\beta-1}\left[1-\left(\sum_{i=1}^{n} p_{i}^{\frac{1}{2-\beta}}\right)^{2-\beta}\right], \quad 0<\beta \leq 1 .
$$

Setting $r=\frac{1}{2-\beta}$ in (1.3), we get

$$
H^{r}(P)=\frac{r}{r-1}\left[1-\left(\sum_{i=1}^{n} p_{i}^{r}\right)^{\frac{1}{r}}\right], \quad \frac{1}{2}<r \leq 1 .
$$

which is a measure mentioned by Arimoto [3] as an example of a generalized class of information measure. It may be marked that (1.4) also approaches to Shannon's entropy as $r \rightarrow 1$.

Hooda-Anant [2] studied (1.2) as a generalization of Shannon's and Bockee-Lubbe [5] using infimum operation.

In the present communication, our main endeavour is to generalize (1.1) as $\alpha$-R-norm information measure as:

$$
H_{R}^{\alpha}(P)=\frac{R}{R-\alpha}\left[1-\left(\sum_{i=1}^{n} p_{i}^{\frac{R}{\alpha}}\right)^{\frac{\alpha}{R}}\right], \quad 0<\alpha \leq 1, R(>0) \neq 1 .
$$

Naturally (1.5) reduces to (1.1).

Measure (1.5) is a non-additive measure which by applying infimum operation is characterized in Section (2) and axiomatically through functional equation in Section (3). Algebraic and analytical properties are studied in Section (4), Section (5) deals with for joint and conditional generalized $\alpha$-R-norm information measrue for joint and conditional distribution.

\section{Characterization}

Applying Infimum Operation

In this section, we consider the $\alpha$-R-norm information measure (1.5) as weighted arithmetic mean representation of elementary R-norm entropies of $\alpha$ of occurrences of various single outcomes.

Theorem 1. Let

$$
\begin{aligned}
f_{R}^{\alpha}\left({ }^{*} P_{i}\right) & =\frac{R}{R-\alpha}\left[1-{ }^{*} p_{i}^{\frac{R-\alpha}{R}}\right], \quad R>0, R \neq 1,0<\alpha \leq 1, \\
\text { then } \quad H_{R}^{\alpha}(P) & =\inf _{{ }^{*} p} \sum_{i=1}^{n} p_{i} f_{R}^{\alpha}\left({ }^{*} p_{i}\right)
\end{aligned}
$$

where the operation infimum is taken over the probability distribution

$$
\left({ }^{*} p_{1},{ }^{*} p_{2}, \ldots,{ }^{*} p_{n}\right) \in \Delta_{n} \text {. }
$$


Proof. Let us consider

$$
\sum_{i=1}^{n} p_{i} f_{R}^{\alpha}\left({ }^{*} p_{i}\right)=\frac{R}{R-\alpha}\left[\sum_{i=1}^{n} p_{i}\left(1-{ }^{*} p_{i}^{\frac{R-\alpha}{R}}\right)\right] .
$$

We minimize (2.3) subject to the natural constraint

$$
\sum_{i=1}^{n}{ }^{*} p_{i}=1
$$

For this we consider Lagrangian

$$
L=\frac{R}{R-\alpha}\left[1-\sum_{i=1}^{n} p_{i}{ }^{*} p_{i}^{\frac{R-\alpha}{R}}\right]+\lambda\left[\sum_{i=1}^{n}{ }^{*} p_{i}-1\right] .
$$

Differentiating (2.5) w.r.t. ${ }^{*} p_{i}$, we have

$$
\frac{\partial L}{\partial^{*} p_{i}}=-p_{i} \cdot{ }^{*} p_{i}^{\frac{\alpha}{R}}+\lambda
$$

For extreme value, we put (2.6) equal to zero which gives

$$
{ }^{*} p_{i}=\frac{\lambda^{-\frac{R}{\alpha}}}{p_{i}^{-\frac{R}{\alpha}}}
$$

We mark here that

$$
\begin{aligned}
& \frac{\partial^{2} L}{\partial^{*} p_{i}^{2}}>0, \\
& \text { when } \quad p_{i}=\frac{\lambda^{-\frac{R}{\alpha}}}{p_{i}^{-\frac{R}{\alpha}}}, \quad \frac{\partial^{2} L}{\partial^{*} p_{i}^{2}}>0 .
\end{aligned}
$$

Hence the value of * $p_{i}$ given by (2.7) is minimum and using (2.4) in (2.7), we can find the value of $\lambda$ and consequently, we have

$$
{ }^{*} p_{i}=\frac{p_{i}^{\frac{R}{\alpha}} \cdot}{\sum_{i=1}^{n} p_{i} \cdot \frac{R}{\alpha}}, \quad 0<\alpha \leq 1, R(>0) \neq 1 .
$$

Now we consider RHS of (2.2)

$$
\begin{aligned}
\left.\inf _{{ }^{*} p_{i}} \sum_{i=1}^{n} p_{i} f_{R}^{\alpha}{ }^{*} p_{i}\right) & =\frac{R}{R-\alpha}\left[1-\sum_{i=1}^{n} p_{i}{ }^{*} p_{i}^{\frac{R-\alpha}{R}}\right] \\
& =\frac{R}{R-\alpha}\left[1-\frac{\sum_{i=1}^{n} p_{i}^{\frac{R}{\alpha}}}{\left(\sum_{i=1}^{n} p_{i}^{\frac{R}{\alpha}}\right)^{\frac{R-\alpha}{R}}}\right]
\end{aligned}
$$




$$
\begin{aligned}
& =\frac{R}{R-\alpha}\left[1-\left(\sum_{i=1}^{n} p_{i}^{\frac{R}{\alpha}}\right)^{\frac{\alpha}{R}}\right] \\
& =H_{R}^{\alpha}(P) .
\end{aligned}
$$

Further without loss of generality, we may assume that corresponding to the observed probability distribution $P \in \Delta_{n}$, there is a prior probability distribution $Q \in \Delta_{n}$ and replacing $f_{R}^{\alpha}\left(^{*} p_{i}\right)$ by $f_{R}^{\alpha}\left(q_{i}\right)$ in (2.2), we have

$$
H_{R}^{\alpha}(P)=\inf _{q_{i}} \sum_{i=1}^{n} p_{i} f_{R}^{\alpha}\left(q_{i}\right) .
$$

In case we do not apply the operation of infimum to (2.9), then it depends on two probability distributions $P$ and $Q$. For $R=1, f_{R}^{\alpha}(q)$ is an analogue of $\frac{1}{1-\alpha}\left(1-q^{1-\alpha}\right)$ which reduces to $\log 1 / q$ when $\alpha \rightarrow 1$. Thus (2.9) is equal to

$$
\frac{1}{\alpha-1}\left(\sum_{i=1}^{n} p_{i} q_{i}^{1-\alpha}-1\right)
$$

which is a generalized inaccuracy measure of degree $\alpha$.

(2.10) reduces to inaccruacy measure of degree $\alpha$ characterized by Sharma-Taneja [10]. Therefore, there is no harm to represents (2.10) through $f_{R}^{\alpha}(q)$.

$$
\begin{aligned}
H_{R}^{\alpha}(P ; Q) & =\sum_{i=1}^{n} p_{i} f_{R}^{\alpha}\left(q_{i}\right) \\
& =\frac{R}{R-\alpha}\left[\sum_{i=1}^{n} p_{i}\left(1-q_{i}^{\frac{R-\alpha}{R}}\right)\right], \quad R(>0) \neq 1,0<\alpha \leq 1 .
\end{aligned}
$$

Infact (2.11) can also be described as the average of elementary $\alpha$-R-norm inaccuracies $f_{R}^{\alpha}\left(q_{i}\right)$, $i=1,2, \ldots, n$ and so called $\alpha$-R-normed inaccuracy measure of degree $\alpha$. Thus it seems plausible that (2.11) may be characterized and then by taking its infimum we can arrive at (1.5).

In the following theorem, we characterize the elementary information function $f_{R}^{\alpha}(q)$ by assuming only two axioms and applying infimum operation.

Theorem 2. Let $f$ be a real valued continuous self-information function defined on $(0,1]$ satisfying the following axioms

Axiom $\mathbf{A}_{1} \cdot f(x, y)=f(x)+f(y)-\frac{R-\alpha}{R} f(x) f(y)$.

Axiom $\mathbf{A}_{2} \cdot f\left(\frac{1}{n}\right)=\frac{R}{R-\alpha}\left(1-n^{\frac{R-\alpha}{R}}\right), R(>0) \neq 1,0<\alpha \leq 1$

and $n=2,3, \ldots$ is maximality constant.

Then $f_{R}^{\alpha}(q)$ defined in (2.9) holds.

Proof. Considering $f(x)=\frac{R}{R-\alpha}(1-\phi(x))$ in axiom $\mathrm{A}_{1}$, we get

$$
\frac{R}{R-\alpha}[1-\phi(x y)]=\frac{R}{R-\alpha}[1-\phi(x)]+\frac{R}{R-\alpha}[1-\phi(y)]
$$


or,

$$
-\frac{R}{R-\alpha}[(1-\phi(x))(1-\phi(y))]
$$

$$
\phi(x y)=\phi(x) \phi(y) .
$$

But the relation (2.12) is a well known Cauchy's functional equation (refer Aczel [1]). The continuous solution of (2.12) is given by $\phi(x)=x^{a}$, where $a \neq 0$ is an arbitrary constant.

On using axiom $\mathrm{A}_{2}$, we get $a=\frac{R-\alpha}{R}$ and hence

$$
f(x)=\frac{R}{R-\alpha}\left[1-x^{\frac{R-\alpha}{R}}\right]
$$

which is exactly of the form of (1.1).

Next, the measure (1.5) can be easily obtained by applying infimum operation on the equation (2.11) on the lines of the Theorem I.

Remarks. For an incomplete probability distribution scheme

$$
P=\left(p_{1}, p_{2}, \ldots, p_{n}\right), \quad p_{i} \geq 0, \quad \sum_{i=1}^{n} p_{i}=1, \quad f_{R}^{\alpha}\left(q_{i}\right), i=1,2, \ldots, n,
$$

associated with individual events may be worked out. Then as in case (2.10), we may define

$$
H_{R}^{\alpha}\left(p_{1}, p_{2}, \ldots, p_{n} ; q_{1}, q_{2}, \ldots, q_{n}\right)=\frac{\sum_{i=1}^{n} p_{i} f_{R}^{\alpha}\left(q_{i}\right)}{\sum_{i=1}^{n} p_{i}}
$$

By using infimum operation with respect to $q_{i}$ 's the equation (2.13) gives

$$
H_{R}^{\alpha}(P)=\frac{R}{R-\alpha}\left[1-\left\{\frac{\sum_{i=1}^{n} p_{i}^{\frac{R}{\alpha}}}{\sum_{i=1}^{n} p_{i}}\right\}\right]
$$

which is the $\alpha$-R-norm entropy of degree $\alpha$ of incomplete probability distribution.

It is also worth mentioning that if we take arithmetic average with weights as continuous function $w$ ( ), then we get the general expression

$$
H_{R}^{\alpha}(P ; Q)=\frac{\sum W\left(p_{i}\right) f_{R}^{\alpha}\left(q_{i}\right)}{\sum_{i=1}^{n} W\left(p_{i}\right)} .
$$

By considering different weight $w$ ( ) satisfying the condition

$$
W(p q)=W(p) W(q) \text {, where } W() \neq 0,
$$

we can obtain various generalized $\alpha$-R-normed information measures. 


\section{Axiomatic characterization of $\alpha$-R-norm information measure}

In this section, we attempt to characterize (2.11) by considering it as inaccuracy measure for two distributors $P$ and $Q \in \Delta_{n}$ and arrive at (1.5) by applying infimum operation with respect to $q_{i}$ 's, $i=1,2, \ldots, n$.

Let $S_{n}=\Delta_{n} \times \Delta_{n} \rightarrow R, n=2,3, \ldots$ and $G_{n}$ be a sequence of functions of $p_{i}$ 's and $q_{i}$ 's, $i=$ $1,2, \ldots, n$ over $S_{n}$ satisfying the following axioms.

Axiom 1. $G_{n}(P, Q)=a_{1}+a_{2} \sum_{i=1}^{n} h\left(p_{i} q_{i}\right)$, where $a_{1}$ and $a_{2}$ are non-zero constants and

$$
p_{i} q_{i} \in j=(0,1) \times(0,1) \cup\{(0, y): 0<y \leq 1\} \cup\left\{\left(1, y^{\prime}\right) ; 0<y^{\prime} \leq 1\right\}
$$

Axiom 2. For $P, Q \in \Delta_{n}$ and $P^{\prime} Q^{\prime} \in \Delta_{m}, G_{n}$ satisfies the following property

$$
G_{n m}\left(P P^{\prime}, Q Q^{\prime}\right)=G_{n}(P, Q)+G_{m}\left(P^{\prime}, Q^{\prime}\right)-\frac{1}{a_{1}} G_{n}(P, Q) G_{m}\left(P^{\prime}, Q^{\prime}\right)
$$

Axiom 3. $h(p, q)$ is a continuous function of its arguments $p$ and $q$.

Axiom 4. Let all $p_{i}^{\prime} s$ and $q_{i}^{\prime} s$ are equiprobable posterior and prior probabilities of events, respectively then

$$
G_{n}\left(\frac{1}{n}, \frac{1}{n}, \ldots \frac{1}{n} ; \frac{1}{n}, \frac{1}{n}, \ldots \frac{1}{n}\right)=\frac{R}{R-\alpha}\left[1-n^{\frac{\alpha-R}{R}}\right],
$$

where $n=2,3, \ldots, R(>0) \neq 1$ and $0<\alpha \leq 1$.

Theorem 3. The inaccuracy measure (2.11) is uniquely determined by the Axioms 1 to 4.

First of all, we prove the following three lemmas to facilitate the proof of the theorem:

Lemma 1. By Axioms 1-2, we have

$$
\sum_{i=1}^{n} \sum_{j=1}^{m} h\left(p_{i} p_{j}^{\prime} ; q_{i} q_{j}^{\prime}\right)=\left(-\frac{a_{2}}{a_{1}}\right) \sum_{i=1}^{n} h\left(p_{i}, q_{i}\right) \sum_{j=1}^{n} h\left(p_{j}^{\prime} ; q_{j}^{\prime}\right)
$$

where $\left(p_{i}, q_{i}\right),\left(p_{j}^{\prime}, q_{j}^{\prime}\right) \in j$ for $i=1,2, \ldots, n$ and $j=1,2, \ldots, m$.

Lemma 2. The continuous solution that satisfies (3.1) is the continuous solution of the functional equation

$$
h\left(p p^{\prime}, q q^{\prime}\right)=\left(-\frac{a_{2}}{a_{1}}\right) h(p, q) h\left(p^{\prime}, q^{\prime}\right)
$$

Proof. Let $a, b, c, d$ and $a^{\prime}, b^{\prime}, c^{\prime}, d^{\prime}$ be positive integers such that $1 \leq a^{\prime} \leq a, 1 \leq b^{\prime} \leq b$, $1 \leq c^{\prime} \leq c$ and $1 \leq d^{\prime} \leq d$.

Setting 


$$
n=a-a^{\prime}+1=b-b^{\prime}+1
$$

and

$$
\begin{aligned}
& m=c-c^{\prime}+1=d-d^{\prime}+1 \\
& p_{i}=\frac{1}{a}\left(i=1,2, \ldots, a-a^{\prime}\right), p_{a-a^{\prime}+1}=\frac{a^{\prime}}{a}, \\
& q_{i}=\frac{1}{b}\left(i=1,2, \ldots, b-b^{\prime}\right), q_{b-b^{\prime}+1}=\frac{b^{\prime}}{b}, \\
& p_{j}^{\prime}=\frac{1}{c}\left(j=1,2, \ldots, c-c^{\prime}\right), p_{c-c^{\prime}+1}=\frac{c^{\prime}}{c}, \\
& q_{j}^{\prime}=\frac{1}{d}\left(j=1,2, \ldots, d-d^{\prime}\right), p_{d-d^{\prime}+1}=\frac{d^{\prime}}{d},
\end{aligned}
$$

in equation (3.1), we have

$$
\begin{gathered}
\left(a-a^{\prime}\right)\left(c-c^{\prime}\right) h\left(\frac{1}{a c}, \frac{1}{b d}\right)+\left(c-c^{\prime}\right) h\left(\frac{a^{\prime}}{a c}, \frac{b^{\prime}}{b d}\right)+\left(a-a^{\prime}\right) h\left(\frac{c^{\prime}}{a c}, \frac{d^{\prime}}{b d}\right)+h\left(\frac{a^{\prime} c^{\prime}}{a c}, \frac{b^{\prime} d^{\prime}}{b d}\right) \\
=\left(-\frac{a_{2}}{a_{1}}\right)\left[\left(a-a^{\prime}\right) h\left(\frac{1}{a}, \frac{1}{b}\right)+h\left(\frac{a^{\prime}}{a}, \frac{b^{\prime}}{b}\right)\right] \times\left[\left(c-c^{\prime}\right) h\left(\frac{1}{c}, \frac{1}{d}\right)+h\left(\frac{c^{\prime}}{c}, \frac{d^{\prime}}{d}\right)\right]
\end{gathered}
$$

taking $a^{\prime}=b^{\prime}=c^{\prime}=d^{\prime}=1$ in (3.3), we get

$$
h\left(\frac{1}{a c}, \frac{1}{b a}\right)=\left(-\frac{a_{2}}{a_{1}}\right) h\left(\frac{1}{a}, \frac{1}{b}\right) h\left(\frac{1}{c}, \frac{1}{d}\right) .
$$

Taking $a^{\prime}=b^{\prime}=1$ in (3.3) and using (3.4), we have

$$
h\left(\frac{c^{\prime}}{a c}, \frac{d^{\prime}}{b d}\right)=\left(-\frac{a_{2}}{a_{1}}\right) h\left(\frac{1}{a}, \frac{1}{b}\right) h\left(\frac{c^{\prime}}{c}, \frac{d^{\prime}}{d}\right) .
$$

Again taking $c^{\prime}=d^{\prime}=1$ in (3.3), we have

$$
h\left(\frac{a^{\prime}}{a c}, \frac{b^{\prime}}{b d}\right)=\left(-\frac{a_{2}}{a_{1}}\right) h\left(\frac{1}{c}, \frac{1}{d}\right) h\left(\frac{a^{\prime}}{a}, \frac{b^{\prime}}{b}\right) .
$$

Now (3.3) together with (3.4), (3.5) and (3.6) reduces to

$$
h\left(\frac{a^{\prime} b^{\prime}}{a b}, \frac{c^{\prime} d^{\prime}}{b d}\right)=\left(-\frac{a_{2}}{a_{1}}\right) h\left(\frac{a^{\prime}}{a}, \frac{b^{\prime}}{b}\right) h\left(\frac{c^{\prime}}{c}, \frac{d^{\prime}}{d}\right) .
$$

Setting $\frac{a^{\prime}}{a}=p, \frac{b^{\prime}}{b}=q, \frac{c^{\prime}}{c}=p^{\prime}, \frac{d^{\prime}}{d}=q^{\prime}$ in (3.7), we get the required result (3.2) for rational numbers which by continuting of $h$ holds for all real $p, q, p^{\prime}, q^{\prime} \in J$.

In the next Lemma, we get the most general solution of (3.2).

Lemma 3. The most general continuous solutions of equation (3.2) are given by

$$
h(p, q)=\left(-\frac{a_{1}}{a_{2}}\right) p^{u} q^{v}, u \neq 0, v \neq 0 .
$$


and

$$
h(p, q)=0
$$

Proof. Taking $g(p, q)=\left(-\frac{a_{2}}{a_{1}}\right) h(p, q)$ in (3.2), we have

$$
g\left(p p^{\prime}, q q^{\prime}\right)=g(p, q) g\left(p^{\prime}, q^{\prime}\right)
$$

The most general continuous solution of (3.10) c.f. Aczel [1] is given by

$$
g(p, q)=p^{u} q^{v}, u \neq 0, v \neq 0
$$

and

$$
g(p, q)=0
$$

On substituting $g(p, q)=\left(-\frac{a_{1}}{a_{1}}\right) h(p, q)$ in (3.11) and (3.12), we get (3.8) and (3.9) respectively. This proves the Lemma 3.

Proof of theorem. Substituting the solution (3.8) in Axiom 1, we have

$$
G_{n}(P, Q)=a_{1}\left(1-\sum_{i=1}^{n} p_{i}^{u} q_{i}^{\nu}\right), u, v \neq 0
$$

Using Axiom 4 in (3.13), we get

$$
a_{1}=\frac{R}{R-\alpha}, u=1 \text { and } v=\frac{R-\alpha}{R} .
$$

Substituting these values in (3.13), we have

$$
G_{n}(P, Q)=H_{R}^{\alpha}(P, Q)
$$

and hence this completes the proof of the Theorem 3.

Remark. In this equation (3.8) if $u=0$ and $v=0$ then $h(p, q)=\left(-\frac{a_{1}}{a_{2}}\right)$ which is a trivial solution and is of no interest. The solution (3.9) does not even contain any variable and hence it is again discarded.

\section{Properties of $H_{R}^{\alpha}(P)$}

This section presents the algebraic and analytical properties of the $\alpha$-R-norm information measure $H_{R}^{\alpha}(P)$ and satisfies the following properties.

1. $H_{R}^{\alpha}(P)=H_{R}^{\alpha}\left(p_{1}, p_{2}, \ldots, p_{n}\right)$ is a symmetric function of $\left(p_{1}, p_{2}, \ldots, p_{n}\right)$.

2. $H_{R}^{\alpha}(P)$ is expansible i.e. $H_{R}^{\alpha}\left(p_{1}, p_{2}, \ldots, p_{n}, 0\right)=H_{R}^{\alpha}\left(p_{1}, p_{2}, \ldots, p_{n}\right)$.

3. $H_{R}^{\alpha}(P)$ is decisive i.e. $H_{R}^{\alpha}(1,0)=H_{R}^{\alpha}(0,1)=0$. 
4. $H_{R}^{\alpha}(P)$ is non-recursive.

5. $H_{R}^{\alpha}(P, Q)=H_{R}^{\alpha}(P)+H_{R}^{\alpha}(Q)-\frac{R-\alpha}{R} H_{R}^{\alpha}(P) H_{R}^{\alpha}(Q)$ i.e. $H_{R}^{\alpha}(P)$ is non-additive.

Proof. Properties 1 to 3 can be verified easily, now we consider 4.

$$
H_{R}^{\alpha}\left(\frac{p_{1}}{p_{1}+p_{2}}, \frac{p_{2}}{p_{1}+p_{2}}\right)=\frac{R}{R-\alpha}\left[1-\left\{\frac{p_{1}^{\frac{R}{\alpha}}+p_{2}^{\frac{R}{\alpha}}}{\left(p_{1}+p_{2}\right)^{\frac{R}{\alpha}}}\right\}^{\frac{\alpha}{R}}\right]
$$

and

$$
H_{R}^{\alpha}\left(p_{1}+p_{2}, p_{3}, \ldots, p_{n}\right)=\frac{R}{R-\alpha}\left[1-\left\{\left(p_{1}+p_{3}\right)^{\frac{R}{\alpha}}+p_{3}^{\frac{R}{\alpha}}+\cdots+p_{n}^{\frac{R}{\alpha}}\right\}^{\frac{\alpha}{R}}\right] .
$$

By combining (4.1) and (4.2), we have

$$
H_{R}^{\alpha}\left(p_{1}+p_{2}, p_{3}, \ldots, p_{n}\right)+\left(p_{1}+p_{2}\right) H_{R}^{\alpha}\left(\frac{p_{1}}{p_{1}+p_{2}}, \frac{p_{2}}{p_{1}+p_{2}}\right) \neq H_{R}^{\alpha}\left(p_{1}, p_{2}, \ldots, p_{n}\right) .
$$

Thus $H_{R}^{\alpha}\left(p_{1}, p_{2}, \ldots, p_{n}\right)$ is non-recursive.

Property 5. Let $A_{1}, A_{2}, \ldots, A_{n}$ and $B_{1}, B_{2}, \ldots, B_{m}$ be the two sets of events associated with probability distributions $P \in \Delta_{n}$ and $Q \in \Delta_{m}$. We denote the probability of the joint occurrence of events

$$
A_{i}=(i=1,2, \ldots, n) \text { and } B_{j}=(j=1,2, \ldots, m) \text { on } p\left(A_{i} \cap B_{j}\right) .
$$

Then the $\alpha$-R-norm entropy is given by

$$
H_{R}^{\alpha}(P * Q)=\frac{R}{R-\alpha}\left[1-\left\{\sum_{i=1}^{n} \sum_{j=1}^{m} p_{i}^{\frac{R}{\alpha}}\left(A_{i} \cap B_{i}\right)\right\}^{\frac{\alpha}{R}}\right] .
$$

Since the events considered here are stochastically independent therefore, we have

$$
\begin{aligned}
H_{R}^{\alpha}(P * Q) & =\frac{R}{R-\alpha}\left[1-\left\{\sum_{i=1}^{n} p_{i}^{\frac{R}{\alpha}}\left(A_{i}\right)\right\}^{\frac{\alpha}{R}}\left\{\sum_{j=1}^{m} p_{j}^{\frac{R}{\alpha}}\left(B_{j}\right)\right\}^{\frac{\alpha}{R}}\right] \\
& =\frac{R}{R-\alpha}\left[1-\left\{\sum_{i=1}^{n} p_{i}^{\frac{R}{\alpha}}\right\}^{\frac{\alpha}{R}}\left\{\sum_{j=1}^{m} p_{j}^{\frac{R}{\alpha}}\right\}^{\frac{\alpha}{R}}\right] \\
& =\frac{R}{R-\alpha}-\frac{R}{R-\alpha}\left[\left(1-\frac{R-\alpha}{R} H_{R}^{\alpha}(P)\right)\left(1-\frac{R-\alpha}{R} H_{R}^{\alpha}(Q)\right)\right] \\
& =H_{R}^{\alpha}(P)+H_{R}^{\alpha}(Q)-\frac{R-\alpha}{R} H_{R}^{\alpha}(P) H_{R}^{\alpha}(Q)
\end{aligned}
$$

Corollary. The Property 5, can also be extended for $m$ stochastically independent distributors $P_{0}, P_{1}, \ldots, P_{m-1}$ having $n_{0}, n_{1}, \ldots, n_{m-1}$ elements respectively. Let

$$
H_{R}^{\alpha}\left(P_{0}\right)=\cdots=H_{R}^{\alpha}\left(P_{m-1}\right)=w .
$$


Then

$$
H_{R}^{\alpha}\left(P^{*}\right)=\frac{R}{R-\alpha}\left[1-\left\{1-\frac{R-\alpha}{R} w\right\}^{m}\right]
$$

where $P^{*}$ stands for the product space of $m$ stochastically independent distributor $P_{0}, P_{1}, \ldots, P_{m-1}$.

Proof. In (4.3), we have proved the results for product of two probability distributors on similar lines, it is very easy to show that

$$
\begin{aligned}
H_{R}^{\alpha}\left(P_{0} P_{1} P_{2}\right)= & H_{R}^{\alpha}\left(P_{0}\right)+H_{R}^{\alpha}\left(P_{1}\right)+H_{R}^{\alpha}\left(P_{2}\right) \\
& -\frac{R-\alpha}{R}\left[H_{R}^{\alpha}\left(P_{0}\right) H_{R}^{\alpha}\left(P_{1}\right)+H_{R}^{\alpha}\left(P_{0}\right) H_{R}^{\alpha}\left(P_{2}\right)+H_{R}^{\alpha}\left(P_{1}\right) H_{R}^{\alpha}\left(P_{2}\right)\right] \\
& +\left(\frac{R-\alpha}{R}\right) H_{R}^{\alpha}\left(P_{0}\right) H_{R}^{\alpha}\left(P_{1}\right) H_{R}^{\alpha}\left(P_{2}\right) .
\end{aligned}
$$

Further, by mathematical Induction, we arrive at

$$
\begin{aligned}
H_{R}^{\alpha}\left(P^{*}\right)= & H_{R}^{\alpha}\left(P_{0}\right)+H_{R}^{\alpha}\left(P_{1}\right)+\cdots+H_{R}^{\alpha}\left(P_{m-1}\right) \\
& -\frac{R-\alpha}{R}\left[H_{R}^{\alpha}\left(P_{0}\right) H_{R}^{\alpha}\left(P_{1}\right)+H_{R}^{\alpha}\left(P_{0}\right) H_{R}^{\alpha}\left(P_{2}\right)+\cdots\right] \\
& +\frac{R-\alpha}{R}\left[H_{R}^{\alpha}\left(P_{0}\right) H_{R}^{\alpha}\left(P_{1}\right) H_{R}^{\alpha}\left(P_{2}\right)+\cdots\right] \\
& +(-1)^{m-1}\left(\frac{R-\alpha}{R}\right)^{m-1} H_{R}^{\alpha}\left(P_{0}\right) H_{R}^{\alpha}\left(P_{1}\right) \cdots H_{R}^{\alpha}\left(P_{m-1}\right) .
\end{aligned}
$$

Setting $H_{R}^{\alpha}\left(P_{0}\right)=\cdots=H_{R}^{\alpha}\left(P_{m-1}\right)=w$, we have

$$
\begin{aligned}
H_{R}^{\alpha}\left(P^{*}\right)= & m w-\left(\frac{R-\alpha}{R}\right)\left(\begin{array}{c}
m \\
2
\end{array}\right) w^{2}+\left(\frac{R-\alpha}{R}\right)^{2}\left(\begin{array}{c}
m \\
3
\end{array}\right) w^{3} \\
& +(-1)^{m-1}\left(\frac{R-\alpha}{R}\right)^{m-1}\left(\begin{array}{l}
m \\
m
\end{array}\right) w^{n} \\
= & (-1)\left[-\left(\begin{array}{c}
m \\
1
\end{array}\right) w+\left(\begin{array}{c}
m \\
2
\end{array}\right)\left(\frac{R-\alpha}{R}\right) w^{2}-\left(\begin{array}{c}
m \\
3
\end{array}\right)\left(\frac{R-\alpha}{R}\right)^{2} w^{3}\right. \\
& \left.+\cdots+(-1)^{m}\left(\frac{R-\alpha}{R}\right)^{m-1}\left(\begin{array}{c}
m \\
m
\end{array}\right) w^{m}\right] \\
= & -\frac{R}{R-\alpha}\left[1-\left(\begin{array}{c}
m \\
1
\end{array}\right) \frac{R-\alpha}{R} w+\left(\begin{array}{c}
m \\
2
\end{array}\right)\left(\frac{R-\alpha}{R}\right)^{2} w^{2}\right. \\
& \left.-\left(\begin{array}{l}
m \\
3
\end{array}\right)\left(\frac{R-\alpha}{R}\right)^{3} w^{3}+\cdots+(-1)^{m}\left(\begin{array}{l}
m \\
m
\end{array}\right)\left(\frac{R-\alpha}{R}\right)^{m} w^{m}-1\right] \\
= & -\frac{R}{R-\alpha}\left[\left(1-\frac{R-\alpha}{R} w\right)^{m}-1\right] \\
= & \frac{R}{R-\alpha}\left[1-\left(1-\frac{R-\alpha}{R} w\right)^{m}\right], \quad \text { which is (4.4). }
\end{aligned}
$$


This completes the proof of the Property 5.

Theorem 4. Let $H_{R}^{\alpha}(P)=H_{R}^{\alpha}\left(p_{1}, p_{2}, \ldots, p_{n}\right)$ be the generalized $\alpha$-R-norm information measure. Then for $P \in \Delta_{n}$ and $R \in R^{+}$and $0<\alpha \leq 1$, we have

(a) $H_{R}^{\alpha}(P)$ is Non-negative.

(b) $H_{R}^{\alpha}(P) \geq H_{R}^{\alpha}(1,0,0, \ldots, 0)=0$.

(c) $H_{R}^{\alpha}(P) \leq H_{R}^{\alpha}\left(\frac{1}{n}, \frac{1}{n}, \ldots, \frac{1}{n}\right)=\frac{R}{R-\alpha}\left[1-n^{\frac{\alpha-R}{R}}\right]$.

(d) $H_{R}^{\alpha}(P)$ is a monotonic function of $P$.

(e) $H_{R}^{\alpha}(P)$ is continuous at $R \in R^{+}$.

(f) $H_{R}^{\alpha}(P)$ is stable in $p_{i}, i=1,2, \ldots, n$.

(g) $H_{R}^{\alpha}(P)$ is small for small probabilities.

(h) $H_{R}^{\alpha}(P)$ is a concave function for all $p_{i}$.

(i) $\lim _{R \rightarrow \infty} H_{R}^{\alpha}(P)=1-\max \cdot p_{i}$.

Proof. To prove that $H_{R}^{\alpha}(P)$ is non-negative, we consider the following cases:

Case I. When $R>\alpha$ or $\frac{R}{\alpha}>1$ then $p_{i}^{\frac{R}{\alpha}} \leq p_{i} \forall i$

$$
\begin{aligned}
& \Rightarrow \sum_{i=1}^{n} p_{i}^{\frac{R}{\alpha}} \leq \sum_{i=1}^{n} p_{i}=1, \\
& \Rightarrow\left[\sum_{i=1}^{n} p_{i}^{\frac{R}{\alpha}}\right]^{\frac{\alpha}{R}} \leq 1
\end{aligned}
$$

Case II. When $0<R<\alpha$ or $0<\frac{R}{\alpha}<1$ then on the same lines, we have

$$
\left[\sum_{i=1}^{n} p_{i}^{\frac{R}{\alpha}}\right]^{\frac{\alpha}{R}} \geq 1
$$

We know that

$$
\frac{R}{R-\alpha}>0 \text { if } R>\alpha \text { and } \frac{R}{R-\alpha}<0 \text { if } R<\alpha .
$$

Hence from (4.6) and (4.7), we conclude that $H_{R}^{\alpha}(P) \geq 0$.

Property (b) follows if one of the probabilities is equal to 1 and others are equal to zero. Property (c) is Axiom 2.

Also it is noted that the generalized $\alpha$-R-norm information measure is maximal if all probabilities are equal and is minimum if one probability is unit and others are zero.

(d) $H_{R}^{\alpha}(P)$ is monotonic iff $H_{R}^{\alpha}(p, 1-p)$ is non-decreasing on $p \in\left[0, \frac{1}{2}\right]$. 
From (1.5), we have

$$
H_{R}^{\alpha}(p, 1-p)=\frac{R}{R-\alpha}\left[1-\left\{(1-p)^{\frac{R}{\alpha}}+p^{\frac{R}{\alpha}}\right\}^{\frac{\alpha}{R}}\right] .
$$

Let us define the function $G(p)$ by

$$
G(p)=1-\left[(1-p)^{\frac{R}{\alpha}}+p^{\frac{R}{\alpha}}\right]^{\frac{\alpha}{R}}
$$

Then

$$
\frac{d G(p)}{d p} \geq 0 \text { for } R>\alpha
$$

and

$$
\frac{d G(p)}{d p} \geq 0 \text { for } 0<R<\alpha
$$

From (4.8), we note that

$$
\begin{aligned}
& \frac{d}{d p} H_{R}^{\alpha}(p, 1-p)=\left(\frac{R}{R-\alpha}\right) \frac{d G(p)}{d p} \text { which gives } \\
& \frac{d}{d p} H_{R}^{\alpha}(p, 1-p) \geq 0 \text { for } R \in R^{+}, p \in[0,1], 0<\alpha \leq 1
\end{aligned}
$$

Thus $H_{R}^{\alpha}(p, 1-p)$ is a non-decreasing function and hence monotomic.

(e) We know that $\left[\sum_{i=1}^{n} p_{i}^{\frac{R}{\alpha}}\right]^{\frac{\alpha}{R}}$ is continuous for $R \in[0, \infty)$ and $0<\alpha \leq 1$.

Hence, $H_{R}^{\alpha}(P)=\frac{R}{R-\alpha}\left[1-\left(\sum_{i=1}^{n} p_{i}^{\frac{R}{\alpha}}\right)^{\frac{\alpha}{R}}\right]$ is also continuous at

$$
R \in R^{+} \text {where } 0<\alpha \leq 1 \text {. }
$$

(f) It is obvious

$$
H_{R}^{\alpha}(P, 0)=H_{R}^{\alpha}(P)
$$

Also it is easy to see that

$$
L t_{p \rightarrow 1^{+}} H_{R}^{\alpha}(P ; 1-p)=H_{R}^{\alpha}(P, 0) .
$$

(g) From (4.8) it follows that

$$
L t_{p \rightarrow 1^{+}} H_{R}^{\alpha}(P, 1-p)=L t_{p \rightarrow 1^{+}} \frac{R}{R-\alpha}\left[1-\left\{p^{\frac{R}{\alpha}}+(1-p)^{\frac{R}{\alpha}}\right\}^{\frac{\alpha}{R}}\right]=0 .
$$

This proves that $H_{R}^{\alpha}(p)$ is small for small probabilities.

(h) Let us define the concave function. 
Definition. A function of over a set $S$ is said to be concave if for all choices of $x_{1}, x_{2}, \ldots x_{m} \in$ $S$ and for all scalars $\lambda_{1}, \lambda_{2}, \ldots, \lambda_{m}$ such that $\lambda_{i} \geq 0, \sum_{i=1}^{m} \lambda_{i}=1$, the following holders

$$
f\left(\sum_{i=1}^{m} \lambda_{i} x_{i}\right) \geq \sum_{i=1}^{m} \lambda_{i} f\left(x_{i}\right)
$$

Here we consider random variable $x$ taking its values in the set $S=\left(x_{1}, x_{2}, \ldots, x_{m}\right)$ and $r$ probability distributions over $S$ on follows:

$$
P_{k}(x)=\left\{p_{k}\left(x_{1}\right), \ldots, p_{k}\left(x_{m}\right)\right\}: p_{k}\left(x_{i}\right) \geq 0, \sum_{i=1}^{m} p_{k}\left(x_{i}\right)=1, \quad k=1,2, \ldots, r .
$$

Let us define another probability distribution over $S$

$$
P_{0}(x)=\left\{p_{0}\left(x_{1}\right), \ldots, p_{0}\left(x_{m}\right)\right\} \exists \forall i^{\prime} s .
$$

$P_{0}\left(x_{i}\right)=\sum_{k=1}^{r} \lambda_{k} p_{k}\left(x_{i}\right)$, where $\lambda_{k}^{\prime} s$ are non-negative scalars stisfying $\sum_{k=1}^{r} \lambda_{k}=1$ then we have

$$
D=\sum_{k=1}^{r} \lambda_{k} H_{R}^{\alpha}\left(P_{k}\right)-H_{R}^{\alpha}\left(P_{0}\right), \quad R(>0) \neq 1,0<\alpha \leq 1 .
$$

$H_{R}^{\alpha}(P)$ will be concave if $D$ is less than zero for $R(>0) \neq 1$ and $0<\alpha \leq 1$, so we consider

$$
\begin{aligned}
D & =\sum_{k=1}^{r} \lambda_{k} H_{R}^{\alpha}\left(P_{k}\right)-H_{R}^{\alpha}\left(P_{0}\right) \\
& =\sum_{k=1}^{r} \lambda_{k}\left[1-\left\{\sum_{i=1}^{m} p_{k}^{\frac{R}{\alpha}}\left(x_{i}\right)\right\}^{\frac{\alpha}{R}}\right] \frac{R}{R-\alpha}-\frac{R}{R-\alpha}\left[1-\left\{\sum_{i=1}^{m} p_{0}^{\frac{R}{\alpha}}\left(x_{i}\right)\right\}^{\frac{\alpha}{R}}\right] \\
& =\frac{R}{R-\alpha}\left[\left[\sum_{i=1}^{m}\left(\sum_{k=1}^{r} \lambda_{k} p_{k}\left(x_{i}\right)\right)^{\frac{R}{\alpha}}\right]^{\frac{\alpha}{R}}-\left[\sum_{k=1}^{r} \lambda_{k}\left(\sum_{i=1}^{m} p_{k}^{\frac{R}{\alpha}}\left(x_{i}\right)\right)^{\frac{\alpha}{R}}\right]\right] .
\end{aligned}
$$

Now using the inequality $\left[\sum_{k=1}^{r} a_{k} x_{k}\right]^{t} \lessgtr \sum_{k=1}^{r} a_{k} x_{k}^{t}$ according as $t \lessgtr 1$, we have

$$
\left[\sum_{k=1}^{r} \lambda_{k} p_{k}\left(x_{i}\right)\right]^{\frac{R}{\alpha}} \lessgtr\left[\sum_{k=1}^{r} \lambda_{k} p_{k}^{\frac{R}{\alpha}}\left(x_{i}\right)\right] \text { according as } \frac{R}{\alpha} \lessgtr 1 \text {. }
$$

Therefore

$$
\begin{gathered}
{\left[\sum_{i=1}^{m}\left(\sum_{k=1}^{r} \lambda_{k} p_{k}\left(x_{i}\right)\right)^{\frac{R}{\alpha}}\right] \lessgtr\left(\sum_{i=1}^{m} \sum_{k=1}^{r} \lambda_{k} p_{k}^{\frac{R}{\alpha}}\left(x_{i}\right)\right) \text { according as } \frac{R}{\alpha} \lessgtr 1 .} \\
D_{1}=\left[\sum_{i=1}^{m}\left(\sum_{k=1}^{r} \lambda_{k} p_{k}\left(x_{i}\right)\right)^{\frac{R}{\alpha}}\right]^{\frac{\alpha}{R}} \lessgtr\left[\sum_{k=1}^{r} \lambda_{k}\left(\sum_{i=1}^{m} p_{k}^{\frac{R}{\alpha}}\left(x_{i}\right)\right)\right]^{\frac{\alpha}{R}} \text { according as } \frac{R}{\alpha} \lessgtr 1 .
\end{gathered}
$$


Moreover,

$$
\left[\sum_{k=1}^{r} \lambda_{k}\left(\sum_{i=1}^{m} p_{k}^{\frac{R}{\alpha}}\left(x_{i}\right)\right)\right]^{\frac{\alpha}{R}} \lessgtr\left[\sum_{k=1}^{r} \lambda_{k}\left(\sum_{i=1}^{m} p_{k}^{\frac{R}{\alpha}}\left(x_{i}\right)\right)^{\frac{\alpha}{R}}\right]=D_{2} \text { according as } \frac{R}{\alpha} \lessgtr 1 .
$$

Thus $D_{2} \lessgtr D_{1}$ according as $\frac{R}{\alpha} \lessgtr 1$, which implies that $D<0$ in view of the sign of $\frac{R}{R-\alpha}$ according as $\frac{R}{\alpha} \lessgtr 1$.

This proved that $H_{R}^{\alpha}(P)$ is concave function $P$.

(i) We set max $p_{i}=p_{k}$, asuming $m_{0}=1,2, \ldots, R>\alpha$ and $0<\alpha \leq 1$, we find

$$
\left[\sum_{i=1}^{m} p_{i}^{\frac{R}{\alpha}}\right]^{\frac{\alpha}{R}} \leq\left[m_{0} p_{k}^{\frac{R}{\alpha}}\right]^{\frac{\alpha}{R}}=m_{0}^{\frac{\alpha}{R}} p_{k} .
$$

It is also noted that for $R>\alpha$.

$$
\left[\sum_{i=1}^{m} p_{i}^{\frac{R}{\alpha}}\right]^{\frac{\alpha}{R}} \geq p_{k}
$$

Combing (4.15) and (4.16), we get

$$
p_{k} \leq\left\{\sum_{i=1}^{m} p_{i}^{\frac{R}{\alpha}}\right\}^{\frac{\alpha}{R}} \leq m_{0}^{\frac{\alpha}{R}} p_{k}
$$

Taking limits for $R \rightarrow \infty$ in (4.17), we have

$$
\lim _{R \rightarrow \infty}\left\{\sum_{i=1}^{m_{0}} p_{i}^{\frac{R}{\alpha}}\right\}^{\frac{\alpha}{R}}=p_{k}=\max _{i} \cdot p_{i}
$$

and finally

$$
\lim _{R \rightarrow \infty} H_{R}^{\alpha}(P)=\lim _{R \rightarrow \infty}\left[1-\left\{\sum_{i=1}^{m_{0}} p_{i}^{\frac{R}{\alpha}}\right)^{\frac{\alpha}{R}}\right]=1-\max _{i} \cdot p_{i} .
$$

This completes the proof of Theorem 4 .

\section{Joint and conditional generalized $\alpha$-R-nom information measure}

The present section depicts the joing and conditional probability distribution of two random variables $\xi$ and $\eta$ having probability distributors $P$ and $Q$ over the sets $X=\left\{x_{1}, x_{2}, \ldots, x_{n}\right\}$ and $Y=\left\{y_{1}, y_{2}, \ldots, y_{n}\right\}$ respectively. The generalized $\alpha$-R-norm information of the random variables $H_{R}^{\alpha}(\xi)=H_{R}^{\alpha}(P)$ and $H_{R}^{\alpha}(\eta)=H_{R}^{\alpha}(Q)$, where

$$
\begin{aligned}
& p_{i}=P_{r}\left(\xi_{i}=x_{i}\right), \quad i=1,2, \ldots, n \\
& p_{i}=P_{r}\left(\eta_{j}=y_{j}\right), \quad j=1,2, \ldots, m
\end{aligned}
$$

are the probabilities of the possible values of the random variables. Similarly, we consider a two-dimensional discrete random variable $(\xi, \eta)$ with joint probability distribution $\pi=\left(\pi_{11}, \pi_{12}, \ldots, \pi_{1 n}\right)$

$$
\pi_{i j}=P_{r}\left(\xi_{i}=x_{i}, \eta_{j}=y_{j}\right), \quad i=1,2, \ldots, n, j=1,2, \ldots, m
$$


is the joint probability for the values $\left(x_{i}, y_{i}\right)$ of $(\xi, \eta)$.

We shall denote conditional probabilities by $p_{i j}$ and $q_{i j}$ such that $\pi_{i j}=p_{i j} q_{j}=q_{j i} p_{i}$

$$
p_{i}=\sum_{j=1}^{m} \pi_{i j} \text { and } q_{i}=\sum_{j=1}^{n} \pi_{i j}
$$

Definition. The joint $\alpha$-R-norm information measure for $R \in R^{+}$and $0<\alpha \leq 1$ is given by

$$
H_{R}^{\alpha}(\xi, \eta)=\frac{R}{R-\alpha}\left[1-\left\{\sum_{i=1}^{n} \sum_{j=1}^{m} \pi_{i j}\right\}^{\frac{\alpha}{R}}\right] .
$$

It may be seen that $H_{R}^{\alpha}(\xi, \eta)$ is symmetric in $\xi$ and $\eta$. Due to non-additivity property if $\xi$ and $\eta$ are stochastically independent. Then the following holds

$$
H_{R}^{\alpha}(\xi, \eta)=H_{R}^{\alpha}(\xi)+H_{R}^{\alpha}(\eta)-\frac{R-\alpha}{R} H_{R}^{\alpha}(\xi) H_{R}^{\alpha}(\eta) .
$$

Definition. The average conditional $\alpha$-R-norm information of $\eta$ given $\xi$ for $R \in R^{+}$and $0<\alpha \leq 1$ is defined as

$$
{ }^{*} H_{R}^{\alpha}(\eta / \xi)=\frac{R}{R-\alpha}\left[1-\sum_{i=1}^{n} p_{i}\left\{\sum_{j=1}^{m} q_{j i}^{\frac{R}{\alpha}}\right\}^{\frac{\alpha}{R}}\right] .
$$

Or alternatively

$$
{ }^{* *} H_{R}^{\alpha}(\eta / \xi)=\frac{R}{R-\alpha}\left[1-\left\{\sum_{i=1}^{n} p_{i} \sum_{j=1}^{m} q_{j i}^{\frac{R}{\alpha}}\right\}^{\frac{\alpha}{R}}\right] .
$$

The two conditional measures (5.3) and (5.4) differ by the way, the probability $p_{i}$ have been taken. The expression (5.3) is a true mathematical expression over $\xi$, whereas the expression (5.4) is not.

The next theorem, proves three results for conditional $\boldsymbol{\alpha}$-R-norm information measures given by (5.3) and (5.4).

Theorem 5. If $\xi$ and $\eta$ are discrete random variables then for

$$
R \in R^{+} \text {and } 0<\alpha \leq 1
$$

then the following results hold.

(i) ${ }^{*} H_{R}^{\alpha}(\eta / \xi) \leq H_{R}^{\alpha}(\eta)$,

(ii) ${ }^{* *} H_{R}^{\alpha}(\eta / \xi) \leq H_{R}^{\alpha}(\eta)$,

(iii) ${ }^{* *} H_{R}^{\alpha}(\eta / \xi) \leq{ }^{*} H_{R}^{\alpha}(\eta / \xi)$,

(iv) ${ }^{* *} H_{R}^{\alpha}(\eta / \xi) \leq{ }^{*} H_{R}^{\alpha}(\eta / \xi) \leq H_{R}^{\alpha}(\eta)$. 
Proof. We know by [4] that for $\frac{R}{\alpha}>1$.

$$
\left[\sum_{j=1}^{m}\left\{\sum_{i=1}^{n} x_{i j}\right\}^{\frac{R}{\alpha}}\right]^{\frac{\alpha}{R}} \leq\left[\sum_{i=1}^{n}\left\{\sum_{j=1}^{m} x_{i j}^{\frac{R}{\alpha}}\right\}^{\frac{R}{\alpha}}\right]^{\frac{\alpha}{R}}
$$

Setting $x_{i j}=\pi i j \geq 0$ in (5.9), we have

$$
\begin{aligned}
& {\left[\sum_{j=1}^{m}\left\{\sum_{i=1}^{n} x_{i j}\right\}^{\frac{R}{\alpha}}\right]^{\frac{\alpha}{R}} \leq\left[\sum_{i=1}^{n}\left\{\sum_{j=1}^{m} x_{i j}^{\frac{R}{\alpha}}\right\}^{\frac{\alpha}{R}}\right] . } \\
& \text { or } \quad\left[\sum_{j=1}^{m} q_{j}^{\frac{R}{\alpha}}\right]^{\frac{\alpha}{R}} \leq\left[\sum_{i=1}^{n}\left\{\sum_{j=1}^{m}\left(q_{j i} p_{i}\right)^{\frac{R}{\alpha}}\right\}^{\frac{\alpha}{R}}\right] \\
& \Rightarrow 1-\left[\sum_{i=1}^{n} p_{i}\left\{\sum_{j=1}^{m} q_{j i}^{\frac{R}{\alpha}}\right\}^{\frac{\alpha}{R}}\right] \leq 1-\left[\sum_{j=1}^{m} q_{j}^{\frac{R}{\alpha}}\right]^{\frac{\alpha}{R}}
\end{aligned}
$$

Using $\frac{R}{R-\alpha}>0$ as $R>\alpha$ and $0<\alpha \leq 1$.

We find that ${ }^{*} H_{R}^{\alpha}(\eta / \xi) \leq H_{R}^{\alpha}(\eta)$.

On the same line, we can prove that (5.12) holds for

$$
0<R<\alpha \text { and } 0<\alpha \leq 1 .
$$

Hence (5.5) holds for all $R \in R^{+}$and $0<\alpha \leq 1$. The equality sign holds iff $\pi_{i j}$ is separable in the sence $\pi_{i j}=p_{i} q_{j}$.

From Jensen's inequality for $R>\alpha$ and $0<\alpha \leq 1$ we find

$$
\sum_{i=1}^{n} p_{i} q_{j i}^{\frac{R}{\alpha}} \geq\left[\sum_{i=1}^{n} p_{i} q_{j i}\right]^{\frac{R}{\alpha}}=q_{j}^{\frac{R}{\alpha}} .
$$

After summation over $j$ and raising both sides to power $\frac{\alpha}{R}$, we have

$$
\left[\sum_{i=1}^{n} p_{i} \sum_{j=1}^{m} q_{j i}^{\frac{R}{\alpha}}\right]^{\frac{\alpha}{R}} \geq\left[\sum_{j=1}^{m} q_{j}^{\frac{R}{\alpha}}\right]^{\frac{\alpha}{R}}
$$

Using $\frac{R}{R-\alpha}>0$ as $R>\alpha$, we get

$$
{ }^{* *} H_{R}^{\alpha}(\eta / \xi) \leq H_{R}^{\alpha}(\eta) .
$$

Equality holds for all $i, q_{j i}=q_{i}$ which is equivalent to the independent property. For $0<R<$ $\alpha$, the inequality (5.14) reverses. However, in view of $\frac{R}{R-\alpha}<0$ as $0<R<\alpha, 0<\alpha \leq 1$ (5.15) still holds. Hence (5.6) is proved. 
Next for the proof of (5.7), we apply Jensen's inequality and

$$
\left[\sum_{i=1}^{n} p_{i}\left\{\sum_{j=1}^{m} q_{j}^{\frac{R}{\alpha}}\right\}^{\frac{\alpha}{R}}\right] \leq\left[\sum_{i=1}^{n} p_{i} \sum_{j=1}^{m} q_{j i}^{\frac{R}{\alpha}}\right]^{\frac{\alpha}{R}}
$$

for $R>\alpha, 0<\alpha \leq 1$ and

$$
\left[\sum_{i=1}^{n} p_{i}\left\{\sum_{j=1}^{m} q_{j}^{\frac{R}{\alpha}}\right\}^{\frac{\alpha}{R}}\right] \geq\left[\sum_{i=1}^{n} p_{i} \sum_{j=1}^{m} q_{j i}^{\frac{R}{\alpha}}\right]^{\frac{\alpha}{R}}
$$

for $0<R<\alpha, 0<\alpha \leq 1$.

Using the fact $\frac{R}{R-\alpha}>0$ and (5.16),

We have

$$
{ }^{* *} H_{R}^{\alpha}(\eta / \xi) \leq{ }^{*} H_{R}^{\alpha}(\eta / \xi) .
$$

From (5.17) for $\frac{R}{R-\alpha}<0$, we get the same result. Hence (5.7) is proved for all $R \in R^{+}$and $0<\alpha \leq 1$ (5.8) is a combination of (5.7) and (5.5). Hence the Theorem 5 is proved.

Note. In this paper, I have define a new function depending on the parameters $\alpha$ and $R$. My motivation for studing this new function is that it generalize R-norm information measure of a discrete probability distribution introduced by Bockee and Lubbe (1980).

\section{References}

[1] J. Aczel, Lectures on Functional Equations and Their Applications, Academic Press, New York, 1966.

[2] Ram Anant, A study of non-additive generalized measures of 'useful' information and J - divergence, Ph.D. Thesis of C.C.S. University, Meerut (India), 1998.

[3] S. Arimoto, Information theoretical considerations on estimation problems, Information and Control 19(1971), 181-194.

[4] E. F. Beckenbach and R. Bellman, Inequalities, Springer - Verlog, Verlag, New York, 1971.

[5] D. E. Bockee and J. C. A. V. D. Lubbe, The R-norm information measure, Information and Control 45(1980), 136-155.

[6] Z. Daroczy, Generalized information functions, Information and Control 16(1970), 36-51.

[7] J. F. Havrda and F. Charvat, Quantification methods of classification process, The Concept of structural $\alpha$-entropy, Kybernetika 3(1967), 30-35.

[8] A. Renyi, On measures of entropy and information, Proc. Fourth Berkeley Symp. On Stat. and Prob. Univ. of California Press No.1 (1961), 547-561.

[9] C. E. Shannon, A mathematical theory of communication, Bell System Tech. Journ. 27(1948), 379423 and 623-656.

[10] B. D. Sharma and I. J. Taneja, Entropy of type $(\alpha, \beta)$ and other generalized measures in information theory, Metrika 22(1975), 205-215.

Department of Mathematics, Faculty of Education, Mekelle University, p.o.box 3050, Mekelle, Ethiopia. E-mail: drsatish74@rediffmail.com 